\title{
Neutrino mass in flavor dependent gauged lepton model
}

\author{
Takaaki Nomura ${ }^{1, *}$ and Hiroshi Okada ${ }^{2, \dagger}$ \\ ${ }^{1}$ School of Physics, KIAS, Seoul 02455, Korea \\ ${ }^{2}$ Physics Division, National Center for Theoretical Sciences, Hsinchu 300, Taiwan
}

(Received 27 July 2017; published 29 March 2018)

\begin{abstract}
We study a neutrino model introducing an additional nontrivial gauged lepton symmetry where the neutrino masses are induced at two-loop level, while the first and second charged-leptons of the standard model are done at one-loop level. As a result of the model structure, we can predict one massless active neutrino, and there is a dark matter candidate. Then we discuss the neutrino mass matrix, muon anomalous magnetic moment, lepton flavor violations, oblique parameters, and relic density of dark matter, taking into account the experimental constraints.
\end{abstract}

DOI: $10.1103 /$ PhysRevD.97.055044

\section{INTRODUCTION}

A flavor dependent gauge model often plays an important role in explaining flavor specific measurements such as deviation from the standard model (SM) in the semileptonic $B$ decay process $B \rightarrow K^{(*)} \mu^{+} \mu^{-}$reported by LHCb [1,2], lepton flavor violations (LFVs) such as the $\mu \rightarrow e \gamma$ process [3], and the muon anomalous magnetic moment $\left(\Delta a_{\mu}\right)$ at Brookhaven National Laboratory [4]. Along these lines of thought, Ref. [5] has established a flavor dependent model with a larger gauge group, and a smaller group such as a $U(1)$ flavor dependent model can be found as a result of partially breaking the symmetry.

In particular, it is interesting to consider the active neutrino mass matrix with a lepton specific flavor dependent gauge symmetry $U(1)_{L}$ in Ref. [5]. The active neutrino masses are not allowed at the tree level and they should be related to the $U(1)_{L}$ gauge symmetry breaking. Then a radiative seesaw scenario is an attractive candidate to generate the mass, which can be achieved by introducing some exotic particles. Furthermore, we expect predictability in the active neutrino mass due to restriction from the gauge symmetry.

In this paper, we construct a neutrino model based on the lepton flavor symmetry $U(1)_{L}$, where the neutrino mass can be induced at two-loop level [6-44]. We first discuss the case of general charge assignment and the conditions needed to cancel gauge anomalies. Then phenomenological

\footnotetext{
*nomura@kias.re.kr

†macokada3hiroshi@cts.nthu.edu.tw
}

Published by the American Physical Society under the terms of the Creative Commons Attribution 4.0 International license. Further distribution of this work must maintain attribution to the author(s) and the published article's title, journal citation, and DOI. Funded by SCOAP . analysis is carried out by fixing the charge assignment for our particle contents. As a result, we predict one massless active neutrino, and discuss a dark matter candidate calculating relic density. In addition, we discuss lepton flavor violations (LFVs) and muon $g-2$ in the model.

This paper is organized as follows. In Sec. II, we show the mechanism of anomaly cancellations, review our model setup, formulate the Higgs sector, fermion sector including active neutrinos, muon anomalous magnetic moment, lepton flavor violations, and phenomenologies of a dark matter candidate where we show the allowed region of DM mass to satisfy the relic density without conflicting with the direct detection bound. In Sec. III, we have a globally numerical analysis, and investigate the allowed region to satisfy all the data that we will discuss. Finally, we devote Sec. IV to our conclusions.

\section{MODEL SETUP AND PHENOMENOLOGIES}

Here we construct our model with $U(1)_{L}$ symmetry and carry out a phenomenological analysis. In the fermion sector, we introduce three exotic singly charged fermions with different $U(1)_{L}$ charges; $E_{L_{a}}$ and $E_{R_{a}}(a=1,2)$, respectively, have $\frac{1}{2}$ and $x_{a}$, while $E_{3}$, which is a vectorlike fermion, has $-x_{1}-x_{2}$. Notice here that the charge of $E_{3}$ is arbitrary in general, but $x_{a}$ are determined by several anomaly cancellations as discussed below. In the boson sector, we introduce three isospin singlet bosons with nonzero vacuum expectation values (VEVs), and $\varphi_{1}$ and $\varphi_{a}(a=1,2)$, respectively, have 1 and $\frac{1}{2}-x_{a}$. In addition, we introduce a singly charged boson $h^{ \pm}$, a doubly charged boson $k^{ \pm \pm}$, and an isospin singlet (doublet) inert boson $s(\eta)$, and each of them has $U(1)_{L}$ charge of $-\frac{1}{2},-1$, and 0 . $H$ is identified as the SM-like Higgs. Furthermore, we impose $Z_{2}$ odd for new fields, except $\varphi_{1, a}$ and $k^{ \pm \pm}$, in order to forbid the mixing between the SM fermions and exotic 
TABLE I. Field contents of fermions and bosons and their generic charge assignments under $S U(2)_{L} \times U(1)_{Y} \times U(1)_{L} \times Z_{2}$, where $E_{3}$ is a vectorlike singly charged fermion, $a(=1-2)$ are flavor indices, and $x_{a}$ is a nonzero arbitral charge.

\begin{tabular}{|c|c|c|c|c|c|c|c|c|c|c|c|c|c|c|}
\hline & $L_{L_{a}}$ & $L_{L_{3}}$ & $e_{R_{a}}$ & $e_{R_{3}}$ & $E_{L_{a}}$ & $E_{R_{a}}$ & $E_{3}$ & $H$ & $\varphi_{a}$ & $\varphi_{1}$ & $\eta$ & $h^{+}$ & $k^{++}$ & $s_{0}$ \\
\hline$S U(2)_{L}$ & 2 & 2 & 1 & 1 & 1 & 1 & 1 & 2 & 1 & 1 & 2 & 1 & 1 & 1 \\
\hline$U(1)_{Y}$ & $-\frac{1}{2}$ & $-\frac{1}{2}$ & -1 & -1 & -1 & -1 & -1 & $\frac{1}{2}$ & 0 & 0 & $\frac{1}{2}$ & 1 & 2 & 0 \\
\hline$U(1)_{L}$ & $x_{a}$ & -1 & $\frac{1}{2}$ & -1 & $\frac{1}{2}$ & $x_{a}$ & $-x_{1}-x_{2}$ & 0 & $\frac{1}{2}-x_{a}$ & 1 & 0 & $-\frac{1}{2}$ & -1 & 0 \\
\hline$Z_{2}$ & + & + & + & + & - & - & - & + & + & + & - & - & + & - \\
\hline
\end{tabular}

one and to assure the stability of the DM candidate: the neutral component of $\eta$. Thus, the neutrino masses are induced at two-loop level as shown below. These particle contents and their charge assignments are summarized in Table I.

Anomaly cancellation: We explore conditions for anomaly cancellations under the $S U(2)_{L} \times U(1)_{Y} \times U(1)_{L}$ gauge symmetry in Table I, where $\left[U(1)_{L}\right]^{2} U(1)_{Y}$ is automatically zero under these symmetries. Thus, one should consider three types of triangle anomalies, including all the families as follows:

$$
\begin{gathered}
{[S U(2)]^{2} U(1)_{L}: x_{1}+x_{2}-1=0,} \\
{\left[U(1)_{Y}\right]^{2} U(1)_{L}: \frac{1}{2}\left(x_{1}+x_{2}\right)-\frac{1}{2}=0,}
\end{gathered}
$$

$$
\begin{gathered}
{\left[U(1)_{L}\right]^{3}:-\left(x_{1}^{3}+x_{2}^{3}\right)+1=0,} \\
U(1)_{L}:-\left(x_{1}+x_{2}\right)+1=0 .
\end{gathered}
$$

Therefore, one finds the following two conditions:

$$
x_{1}+x_{2}=1, \quad x_{1}^{2}-x_{1} x_{2}+x_{2}^{2}=1 .
$$

One finds several simple solutions such as $\left(x_{1}, x_{2}\right)=$ $(0,1),(1,0)$ that respectively correspond to $\mu-\tau$ and $e-\tau$ symmetry for lepton doublets.

Fixing charge assignments: Here we fix charge assignments of $U(1)_{L}$ to be $x_{1}=0$ and $x_{2}=1$ for simplicity. In this case, one can simply define $\varphi_{a} \equiv \varphi_{0}$ with a $U(1)_{L}$ charge of $\frac{1}{2}$. Under these symmetries in Table I, the renormalizable Lagrangian in the lepton sector and Higgs potential are respectively given by

$$
\begin{gathered}
-\mathcal{L}_{\text {lepton }}=y_{\ell_{3}} \bar{L}_{L_{3}} H e_{R_{3}}+f_{\alpha} \bar{E}_{R_{\alpha}} \tilde{\eta} L_{L_{\alpha}}+g_{L_{a b}} \bar{E}_{L_{a}}^{c} E_{L_{b}} k^{++}+\kappa_{a} \bar{E}_{L_{a}} e_{R_{a}} s_{0} \\
+y_{\varphi_{a 1}} \bar{E}_{L_{a}} E_{R_{1}} \varphi_{0}+y_{\varphi_{a 2}} \bar{E}_{L_{a}} E_{R_{2}} \varphi_{0}^{*}+y_{\varphi_{31}} \bar{E}_{L_{3}} E_{R_{1}} \varphi_{1}^{*}+M_{33} \bar{E}_{L_{3}} E_{R_{3}} \\
+g_{a b} \bar{e}_{R_{a}}^{c} e_{R_{b}} k^{++}+g_{R_{12}}\left(\bar{E}_{R_{1}}^{c} E_{R_{2}}+\bar{E}_{R_{2}}^{c} E_{R_{1}}\right) k^{++}+\text {c.c. }, \\
V_{\text {nontrivial }}=\mu\left[H^{\dagger} \eta s_{0}+\text { c.c. }\right]+\frac{\mu_{k h h}}{2}\left[k^{++}\left(h^{-}\right)^{2}+\text { c.c. }\right]+\frac{\mu_{\varphi}}{2}\left[\varphi_{0}^{2} \varphi_{1}^{*}+\text { c.c. }\right]+\lambda_{0}\left[\left(H^{T} i \sigma_{2} \eta\right) h^{-} \varphi_{0}^{*}+\text { c.c. }\right],
\end{gathered}
$$

where $\alpha$ runs over 1 to 3 , and $(a, b)$ run over 1 to $2, \tilde{H} \equiv$ $\left(i \sigma_{2}\right) H^{*}$ with $\sigma_{2}$ being the second Pauli matrix. The first term gives the masses for the SM charged leptons, and $f, g_{L / R}$ as well as $\mu_{k h h}$ and $\lambda_{0}$ contribute to the structure of neutrino masses. $\mu_{\varphi}$ forbids the massless goldstone boson (GB) arising from $\varphi_{0,1}$. On the other hand, $g_{a b}$ does not contribute to the neutrino masses and $g_{R_{12}}$ itself cannot reproduce the experimental results for neutrinos. Thus, we just neglect these terms in our analysis.

Next we formulate the scalar sector, in which we parametrize as follows:

$H=\left[\begin{array}{c}w^{+} \\ \frac{v+h+i z}{\sqrt{2}}\end{array}\right], \quad \eta=\left[\begin{array}{c}\eta^{+} \\ \frac{\eta_{0}+i \eta_{l}}{\sqrt{2}}\end{array}\right], \quad \varphi_{0,1}=\frac{v_{0,1}^{\prime}+\sigma_{0,1}+i z_{\varphi_{0,1}}^{\prime}}{\sqrt{2}}$, where $w^{+}$, two massless eigenstates among $z$, and $z_{\varphi_{0,1}}^{\prime}$ are absorbed by the SM and $U(1)_{L}$ gauge bosons $W^{+}, Z$, and $Z^{\prime}$. Inserting tadpole conditions, the $C P$-even mass matrix in the basis of $\left(h, \sigma_{0}, \sigma_{1}\right)$ can be formulated by $O_{R} M_{R}^{2} O_{R}^{T} \equiv \operatorname{Diag}\left[m_{h_{1}}^{2}, m_{h_{2}}^{2}, m_{h_{3}}^{2}\right]$, where $h_{1}$ is the SM Higgs and $O_{R}$ is a three by three orthogonal mixing matrix. On the other hand, the inert boson mass matrix in the basis of $\left(\eta_{0}, s_{0}\right)$ is formulated by $U M_{\text {inert }}^{2} U^{T} \equiv \operatorname{Diag}\left[m_{\eta}^{2}, m_{s}^{2}\right]$, where $U$ is a two by two orthogonal matrix. Then one can parametrize the relation between flavor and the mass eigenstate as [22]

$$
\left[\begin{array}{l}
\eta_{0} \\
s_{0}
\end{array}\right]=\left[\begin{array}{cc}
\cos \beta & -\sin \beta \\
\sin \beta & \cos \beta
\end{array}\right]\left[\begin{array}{l}
\eta \\
s
\end{array}\right]
$$


where $\sin \beta$ is proportional to $\mu v$, and we expect $\sin \beta \ll 1$ in our analysis below. On the other hand, the singly charged boson mass matrix in basis of $\left(\eta^{ \pm}, h^{ \pm}\right)$is formulated by $O_{ \pm} M_{ \pm}^{2} O_{ \pm}^{T} \equiv \operatorname{Diag}\left[m_{H_{1}^{ \pm}}^{2}, m_{H_{2}^{ \pm}}^{2}\right]$, where $O_{ \pm}$is two by two orthogonal matrix. ${ }^{1}$ Then one can parametrize the relation between flavor and mass eigenstate as [22]

$$
\left[\begin{array}{l}
\eta^{ \pm} \\
h^{ \pm}
\end{array}\right]=\left[\begin{array}{cc}
\cos \theta & -\sin \theta \\
\sin \theta & \cos \theta
\end{array}\right]\left[\begin{array}{l}
H_{1}^{ \pm} \\
H_{2}^{ \pm}
\end{array}\right],
$$

where $\sin \theta$ is proportional to $\lambda_{0} v v_{0}^{\prime}$.

$Z^{\prime}$ boson: A massive $Z^{\prime}$ boson appears after spontaneous symmetry breaking of $U(1)_{L}$. The mass of $Z^{\prime}$ is given by $m_{Z^{\prime}}=g^{\prime} \sqrt{v_{0}^{\prime 2}+4 v_{1}^{\prime 2}} / 2$ where $g^{\prime}$ is gauge coupling of $U(1)_{L}$. Since the $Z^{\prime}$ couples to electrons, the mass and gauge coupling are constrained by the LEP data. Here we simply assume the mass is around $\mathrm{TeV}$ scale and the value of $g^{\prime}$ satisfies the constraints. Note that $Z^{\prime}$ does not contribute to neutrino mass generation and our DM candidate has no direct interaction with the $Z^{\prime}$ since $\eta$ does not have a $U(1)_{L}$ charge. Thus, we will not discuss $Z^{\prime}$ physics in our analysis.

Exotic charged-fermion masses: After the symmetry breaking, the exotic charged fermion mass matrix in Eq. (2.7) can be given in the basis $\left[E_{1}, E_{2}, E_{3}\right]^{T}$ as follows:

$$
M_{E} \equiv\left[\begin{array}{ccc}
M_{11} & M_{12} & M_{13} \\
M_{12} & M_{22} & 0 \\
M_{13} & 0 & M_{33}
\end{array}\right],
$$

where we have assumed $M_{E}$ to be a real symmetric matrix for simplicity and define $M_{11} \equiv y_{\varphi_{11}} v_{0}^{\prime} / \sqrt{2}$, $M_{12} \equiv y_{\varphi_{21}} v_{0}^{\prime} / \sqrt{2}, \quad M_{22} \equiv y_{\varphi_{22}} v_{0}^{\prime} / \sqrt{2}, \quad$ and $\quad M_{13} \equiv$ $y_{\varphi_{31}} v_{1}^{\prime} / \sqrt{2}$. Then $M_{E}$ is diagonalized by an orthogonal mixing matrix $V\left(V V^{T}=1\right)$ as

$$
V M_{E} V^{T}=D_{E} \equiv \operatorname{Diag}\left[M_{e}, M_{\mu}, M_{\tau}\right], \quad E_{1,2,3}=V^{T} E_{e, \mu, \tau},
$$

where $M_{e, \mu, \tau}$ is the mass eigenstate.

$S M$ charged-fermion masses: Since the first and second charged leptons are not induced at the tree level, they are rather done at the one-loop level in Fig. 1. In order to formulate these masses, let us write down the relevant Lagrangian to the SM charged leptons in the mass eigenbasis as

\footnotetext{
${ }^{1}$ In general, it should be unitary, but it can be orthogonal when all the couplings of the Higgs potential are real.
}

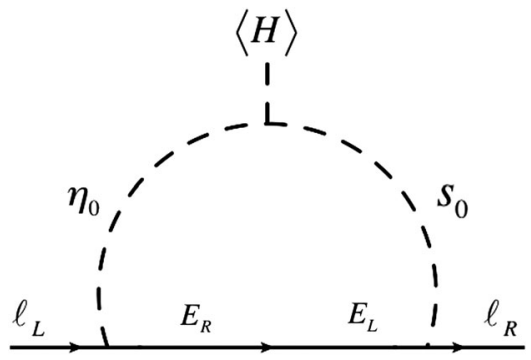

FIG. 1. The one-loop diagram to generate SM charged-lepton masses.

$$
\begin{aligned}
-\mathcal{L} \sim & \sum_{i}^{1-3} \sum_{\alpha}^{e, \mu, \tau} V_{\alpha i} f_{i} \bar{E}_{R_{\alpha}} \ell_{L_{i}}(\cos \beta \eta-\sin \beta s) \\
& +\sum_{j}^{1,2} \sum_{\alpha}^{e, \mu, \tau} V_{\alpha j} \kappa_{j} \bar{E}_{L_{\alpha}} \ell_{R_{j}}(\sin \beta \eta+\cos \beta s)+m_{\ell_{33}} \bar{e}_{L_{3}} e_{R_{3}} \\
\equiv & \sum_{i}^{1-3} \sum_{\alpha}^{e, \mu, \tau} F_{\alpha i} \bar{E}_{R_{\alpha}} \ell_{L_{i}}(\cos \beta \eta-\sin \beta s) \\
& +\sum_{j}^{1,2} \sum_{\alpha}^{e, \mu, \tau} H_{\alpha j} \bar{E}_{L_{\alpha}} \ell_{R_{j}}(\sin \beta \eta+\cos \beta s)+m_{\ell_{33}} \bar{e}_{L_{3}} e_{R_{3}},
\end{aligned}
$$

where $m_{\ell_{33}} \equiv y_{\ell_{3}} v / \sqrt{2}$. Then the mass matrix for the charged leptons can be induced as follows $[36,45,46]$ :

$$
\left(m_{\ell}\right)_{a b}=\sum_{j}^{1,2} \sum_{i}^{1-3}\left(\delta m_{\ell}\right)_{j i}+m_{\ell_{33}}
$$

$$
\begin{aligned}
\left(\delta m_{\ell}\right)_{j i}= & \frac{\sqrt{2} \sin \beta \cos \beta}{(4 \pi)^{2}} \sum_{\alpha}^{e, \mu, \tau}\left(H^{\dagger}\right)_{j \alpha} M_{\alpha} F_{\alpha i} \\
& \times \int_{0}^{1} d x \frac{x M_{\alpha}^{2}+(1-x) m_{s}^{2}}{x M_{\alpha}^{2}+(1-x) m_{\eta}^{2}}
\end{aligned}
$$

$\left(m_{\ell}\right)_{a b}$ is generally diagonalized by biunitary matrices as $V_{\ell_{R}}\left(m_{\ell}\right)_{a b} V_{\ell_{L}}^{\dagger} \equiv D_{\ell}$, where $D_{\ell}$ is the mass eigenstate of charged leptons. Then the resulting mass eigenvalues for the SM charged leptons are generally given by

$$
V_{\ell_{L}} m_{\ell}^{\dagger} m_{\ell} V_{\ell_{L}}^{\dagger}=\operatorname{diag}\left(\left|m_{e}\right|^{2},\left|m_{\mu}\right|^{2},\left|m_{\tau}\right|^{2}\right)
$$

Thus, the observed lepton mixing arises from the neutrino part only.

Active neutrinos: First of all, let us write down the relevant Lagrangian to the neutrinos in the mass eigenbasis as 


$$
\begin{aligned}
-\mathcal{L} & \sim \sum_{\alpha=e, \mu, \tau} V_{\alpha i} f_{i} \bar{E}_{\alpha} P_{L} \nu_{i}\left(\cos \theta H_{1}^{-}-\sin \theta H_{2}^{-}\right)+\sum_{a, b=1}^{2} V_{i a}\left(g_{L}\right)_{a b}\left(V^{T}\right)_{b j} \bar{E}_{L_{i}}^{c} E_{L_{j}} k^{++}+\text {c.c. } \\
& \equiv \sum_{\alpha=e, \mu, \tau} F_{\alpha i} \bar{E}_{\alpha} P_{L} \nu_{i}\left(\cos \theta H_{1}^{-}-\sin \theta H_{2}^{-}\right)+\left(G_{L}\right)_{i j} \bar{E}_{L_{i}}^{c} E_{L_{j}} k^{++}+\text {c.c. }
\end{aligned}
$$

where $g_{L}$ is the rank two matrix. Then the neutrino mass matrix is induced at the two-loop level in Fig. 2, which is given by [22]

$$
\begin{aligned}
\left(m_{\nu}\right)_{i j} \approx & \frac{\mu_{k h h} \sin ^{2} 2 \theta}{2(4 \pi)^{4}} \sum_{\alpha, \beta=e, \mu, \tau}\left[\frac{F_{i \alpha}^{T} M_{\alpha}\left(G_{L}\right)_{\alpha \beta} M_{\beta} F_{\beta j}}{M_{\alpha}^{2}}\right] \\
& \times\left[\mathcal{F}_{I}\left(r_{H_{1}}^{\alpha}, r_{k}^{\alpha}, r_{H_{1}}^{\alpha}, r_{\alpha}^{\beta}\right)-\mathcal{F}_{I}\left(r_{H_{2}}^{\alpha}, r_{k}^{\alpha}, r_{H_{1}}^{\alpha}, r_{\alpha}^{\beta}\right)-\mathcal{F}_{I}\left(r_{H_{1}}^{\alpha}, r_{k}^{\alpha}, r_{H_{2}}^{\alpha}, r_{\alpha}^{\beta}\right)+\mathcal{F}_{I}\left(r_{H_{2}}^{\alpha}, r_{k}^{\alpha}, r_{H_{2}}^{\alpha}, r_{\alpha}^{\beta}\right)\right], \\
& \mathcal{F}_{I}\left(r_{1}, r_{2}, r_{3}, r_{4}\right) \equiv \int_{0}^{1} \frac{[d x]\left[d x^{\prime}\right]}{z(z-1)\left(x^{\prime}+y^{\prime} r_{1}\right)-z^{\prime}\left(x r_{4}+y r_{3}+z r_{2}\right)},
\end{aligned}
$$

where $r_{\beta}^{\alpha} \equiv \frac{M_{\beta}^{2}}{M_{\alpha}^{2}},[d x] \equiv d x d y d z \delta(x+y+z-1)$, and $\left[d x^{\prime}\right] \equiv d x^{\prime} d y^{\prime} d z^{\prime} \delta\left(x^{\prime}+y^{\prime}+z^{\prime}-1\right)$. At the same time, we also formulate the experimental neutrino mass matrix as $m_{\nu}^{\exp } \approx\left|V_{M N S}^{\dagger} D_{\nu} V_{M N S}^{*}\right|$, which can be determined by neutrino oscillation data when numerical (Dirac and Majorana) phases are provided. Notice here that one of the three active neutrino mass eigenstates is massless, since the matrix rank $g_{L}$ is two; $\left|m_{\nu_{1(3)}}\right|=0$ in case of normal(inverted) hierarchy. The structure of the mass matrix indicate that the neutrino mixing angles mainly arise from the mixing matrix $V$. Then one finds the following ranges at $3 \sigma$ confidential level [47] given by, ${ }^{2}$ assuming the normal hierarchy:

$$
m_{\nu}^{\exp } \approx\left[\begin{array}{ccc}
0.0845-0.475 & 0.0629-0.971 & 0.0411-0.964 \\
* & 1.44-3.49 & 1.94-2.85 \\
* & * & 1.22-3.33
\end{array}\right] \times 10^{-11} \mathrm{GeV}
$$

and Majorana phases $\alpha_{1,2}$ taken to be $\alpha_{1,2} \in[-\pi, \pi]$. In the numerical analysis, we impose the constraint $\left|m_{\nu}\right| \approx m_{\nu}^{\exp }$.

Muon anomalous magnetic moment $\left(\Delta a_{\mu}\right): \Delta a_{\mu}$ has been observed and its discrepancy is estimated by [48]

$$
\Delta a_{\mu}=(26.1 \pm 8.0) \times 10^{-10}
$$

The relevant Yukawa Lagrangian contributing to $\Delta a_{\mu}$ as well as LFVs in the mass eigenbasis is given by

$$
-\mathcal{L} \sim \sum_{i}^{1-3} \sum_{\alpha=e, \mu, \tau} F_{\alpha i} \bar{E}_{\alpha} P_{L} \ell_{i} \eta+\sum_{j}^{1,2} \sum_{\alpha}^{e, \mu, \tau} H_{\alpha j} \bar{E}_{L_{\alpha}} \ell_{R_{j}} s+\text { c.c. },
$$

where $\eta \equiv \eta_{R} \simeq \eta_{I}$ and $\sin \beta \ll 1$. Then our $\Delta a_{\mu}$ is induced by interaction with $F$ coupling as explained above, and its form is computed as

\footnotetext{
${ }^{2}$ Recently $\delta=-\pi / 2$ is experimentally favored. However, our result does not change significantly, even if we fix to be $\delta=-\pi / 2$.
}

$$
\begin{aligned}
\Delta a_{\mu}^{Y} \approx & \frac{2 m_{\mu}^{2}}{(4 \pi)^{2}} \sum_{\alpha=e, \mu, \tau}\left(\left|F_{2 \alpha}\right|^{2} F_{I I}\left(m_{\eta}, M_{\alpha}\right)\right. \\
& \left.+\left|H_{2 \alpha}\right|^{2} F_{I I}\left(m_{s}, M_{\alpha}\right)\right),
\end{aligned}
$$

$$
\begin{aligned}
& F_{I I}\left(m_{a}, m_{b}\right) \\
& \quad \equiv \frac{2 m_{a}^{6}+3 m_{a}^{4} m_{b}^{2}-6 m_{a}^{2} m_{b}^{4}+m_{b}^{6}+12 m_{a}^{4} m_{b}^{2} \ln \left[\frac{m_{b}}{m_{a}}\right]}{12\left(m_{a}^{2}-m_{b}^{2}\right)^{4}} .
\end{aligned}
$$

Considering the neutrino oscillations and lepton flavor violations for the $F$ term as will be discussed below, we find the maximal order of $\Delta a_{\mu}^{Y}$ to be $10^{-12}$. On the other hand,

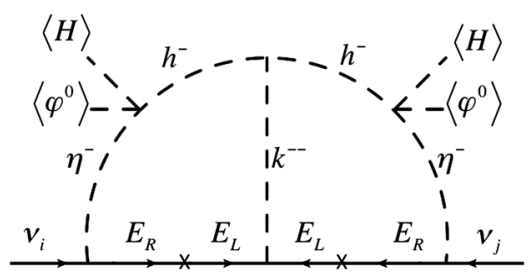

FIG. 2. The two-loop diagram to generate neutrino masses. 
the term with $H_{2 \alpha}$ provides the dominant contribution to $\Delta a_{\mu}$, since it is not constrained by any phenomenologies once we take $H_{21}, H_{23} \ll H_{22}$.

In addition, the $Z^{\prime}$ gauge boson can contribute to $\Delta a_{\mu}$ and its form is approximately given by

$$
\Delta a_{\mu}^{Z^{\prime}} \approx \frac{g_{Z^{\prime}}^{2} m_{\mu}^{2}}{48 \pi^{2} M_{Z^{\prime}}^{2}},
$$

where $Z^{\prime}$ is the new gauge vector boson. Since the righthanded electron couples to the $Z^{\prime}$ boson, we have the constraint $3.7 \mathrm{TeV} \lesssim M_{Z^{\prime}} / g_{Z^{\prime}}$ [49]; the maximal value of $\Delta a_{\mu}^{Z^{\prime}}$ is $\Delta a_{\mu}^{Z^{\prime}}(\operatorname{Max})=1.72 \times 10^{-12}$. Combining $\Delta a_{\mu}^{Y}$ and $\Delta a_{\mu}^{Z^{\prime}}$, we find the final result of muon $g-2, \Delta a_{\mu} \equiv$ $\Delta a_{\mu}^{Y}+\Delta a_{\mu}^{Z^{\prime}}$, where we will adapt the maximal value $\Delta a_{\mu}^{Z^{\prime}}(\operatorname{Max})$ in our numerical analysis below.

Lepton flavor violations: LFV processes of $\ell \rightarrow \ell^{\prime} \gamma$ are given by the same term as the $(g-2)_{\mu}$, and their forms are given by

$$
\begin{aligned}
& \operatorname{BR}\left(\ell_{i} \rightarrow \ell_{j} \gamma\right) \\
& \quad \approx \frac{48 \pi^{3} C_{i j} \alpha_{e m}}{(4 \pi)^{4} G_{F}^{2}}\left|\sum_{\alpha=e, \mu, \tau} F_{j \alpha}^{\dagger} F_{\alpha i} F_{I I}\left(m_{\eta^{0}}, M_{\alpha}\right)\right|^{2},
\end{aligned}
$$

where $\alpha_{e m} \approx 1 / 137$ is the fine-structure constant, $G_{F} \approx$ $1.17 \times 10^{-5} \mathrm{GeV}^{-2}$ is the Fermi constant, and $C_{21} \approx 1$, $C_{31} \approx 0.1784, C_{32} \approx 0.1736$. Experimental upper bounds are given by $[3,50]$ :

$$
\begin{array}{ll}
\operatorname{BR}(\mu \rightarrow e \gamma) \lesssim 4.2 \times 10^{-13}, & \operatorname{BR}(\tau \rightarrow e \gamma) \lesssim 3.3 \times 10^{-8}, \\
\operatorname{BR}(\tau \rightarrow \mu \gamma) \lesssim 4.4 \times 10^{-8}, &
\end{array}
$$

where we define $\ell_{1} \equiv e, \ell_{2} \equiv \mu$, and $\ell_{3} \equiv \tau$.

Oblique parameter: Since we have an isospin doublet boson $\eta$, we have to consider the oblique parameter known as $\Delta S$ and $\Delta T$ [51]. In our case, one finds the following relations:

$$
\begin{aligned}
& \Delta S \approx \frac{1}{12 \pi} \ln \left[\frac{m_{\eta}^{2}}{m_{H_{1}^{ \pm}}^{2}}\right], \\
& \Delta T \approx \frac{1}{16 \pi^{2} v^{2} \alpha_{\mathrm{em}}}\left[\frac{m_{\eta}^{2}+m_{H_{1}^{ \pm}}^{2}}{2}-\frac{m_{H_{1}^{ \pm}}^{2} m_{\eta}^{2}}{m_{H_{1}^{ \pm}}^{2}-m_{\eta}^{2}} \ln \left[\frac{m_{H_{1}^{ \pm}}^{2}}{m_{\eta}^{2}}\right]\right],
\end{aligned}
$$

where we recall the conditions $(\theta, \beta) \ll 1 ; m_{\eta^{ \pm}} \approx m_{H_{1}^{ \pm}}$and $m_{\eta} \equiv m_{\eta_{R}} \approx m_{\eta_{I}}$. Then the current bounds are given by [52]

$$
-0.04 \lesssim \Delta S \lesssim 0.14, \quad 0.01 \lesssim \Delta T \lesssim 0.15 .
$$

Dark matter: In our model, we have two types of DM candidates: $\eta$ and $s$. But let us here focus on the neutral component of $\eta$, resymbolized as a DM candidate by $X \equiv \eta$, because $\eta$ is more testable than $s$ due to constraining the mass from experiments such as neutrino oscillations, LFVs, and oblique parameters. Although the general analysis has been done by Ref. [53], we impose a constraint of the DM mass, which is smaller than the mass of the $W$ boson but greater than half of the $Z$ boson mass, in order to forbid the invisible decay of the $Z$ boson; therefore,

$$
\frac{m_{Z}}{2} \lesssim M_{X} \lesssim m_{W}
$$

This region is favorable for getting sizable muon $g-2$, and well testable in the direct detection constraint such as LUX experiment [54] because it provides the most severe bound at around $50 \mathrm{GeV}$. Under the condition, we have two relevant annihilation cross sections to explain the relic density of DM. One mode arises from Yukawa coupling $F$ that gives the $d$-wave dominance, and another one does from the $s$ channel via SM Higgs with a final state of bottom pairs, where we assume mixing among the $C P$-even neutral bosons is negligible. The $d$-wave dominant cross section given by Eq. (2.24) is found to be [55]

$$
\sigma v_{\text {rel }} \approx \frac{M_{X}^{6}}{60 \pi} \sum_{i, j=1}^{3}\left|\sum_{\alpha=e, \mu, \tau} \frac{F_{i \alpha}^{\dagger} F_{\alpha j}}{\left(M_{\alpha}^{2}+M_{X}^{2}\right)^{2}}\right|^{2} .
$$

In our estimation, however, this cross section reaches $10^{-10}[\mathrm{GeV}]^{-2}$ at most, which is smaller than the cross section required to give the correct relic density by one order of magnitude. Thus, we have to rely on the Higgs portal interaction mode, and its dimensionless cross section $W$ is found to be

$$
\begin{aligned}
& W(s)=\frac{3 \lambda_{X X h_{1}}^{2} m_{b}^{2}\left(s-4 m_{b}^{2}\right)}{4 \pi\left|s-m_{h_{1}}^{2}+i m_{h} \Gamma_{\text {tot }}^{h_{1}}\right|^{2}} \sqrt{1-\frac{4 m_{b}^{2}}{s}}, \\
& \Gamma_{\text {tot }}^{h_{1}} \equiv \Gamma_{\mathrm{SM}}^{h_{1}}+\Gamma_{\text {inv }}^{h}, \\
& \Gamma_{\text {inv }}^{h}=\frac{\mu_{X X h_{1}}^{2} v^{2}}{64 \pi} \sqrt{1-\frac{4 M_{X}^{2}}{m_{h_{1}}}} \Theta\left(\frac{m_{h_{1}}}{2}-M_{X}\right),
\end{aligned}
$$

where $\lambda_{X X h_{1}}\left(\equiv \lambda_{H \eta}+\lambda_{H \eta}^{\prime}+2 \lambda_{H \eta}^{\prime \prime}\right)$ is the trilinear couplings of $X-X-h_{1}$, arising from $\lambda_{H \eta}|\eta|^{2}|H|^{2}+\lambda_{H \eta}^{\prime}\left|\eta^{\dagger} H\right|^{2}+$ $\lambda_{H \eta}^{\prime \prime} / 2\left(\eta^{\dagger} H\right)^{2}+$ H.c. Notice here that $\mu_{X X h_{1}}$ is restricted by the direct detection with spin independent scattering via the Higgs portal, ${ }^{3}$ and its bound is conservatively found to be [55]

\footnotetext{
${ }^{3}$ The constraint of invisible decay of the SM Higgs always gives milder than the one of direct detection in our parameter region. Thus, we will not discuss here.
} 


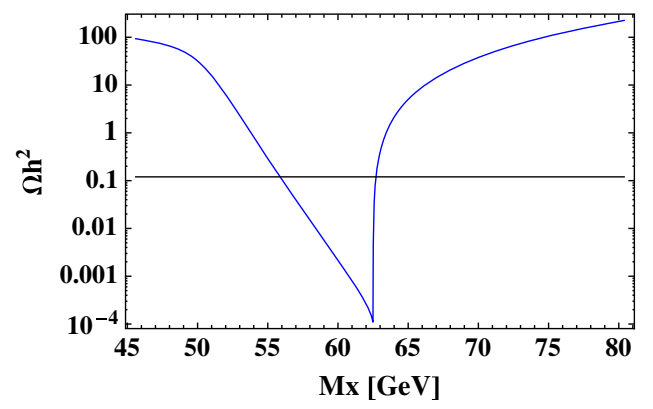

FIG. 3. Plot line of relic density in terms of the DM mass, where we have used $\lambda_{X X h_{1}}^{\mathrm{Max}}=0.01$. The horizontal line represents the measured relic density $\sim 0.12$.

$$
\lambda_{X X h_{1}} \lesssim 0.01
$$

Here we apply the following formula to get the relic density of DM given by [56]:

$$
\Omega h^{2} \approx \frac{1.07 \times 10^{9}}{\sqrt{g_{*}\left(x_{f}\right)} M_{\mathrm{Pl}} J\left(x_{f}\right)[\mathrm{GeV}]},
$$

where $g^{*}\left(x_{f} \approx 25\right)$ is the degrees of freedom for relativistic particles at temperature $T_{f}=M_{X} / x_{f}, M_{\mathrm{Pl}} \approx$ $1.22 \times 10^{19} \mathrm{GeV}$, and $J\left(x_{f}\right)\left(\equiv \int_{x_{f}}^{\infty} d x \frac{\left\langle\sigma v_{\text {rel }}\right\rangle}{x^{2}}\right)$ is given by [57]

$J\left(x_{f}\right)=\int_{x_{f}}^{\infty} d x\left[\frac{\int_{4 M_{X}^{2}}^{\infty} d s \sqrt{s-4 M_{X}^{2}} W(s) K_{1}\left(\frac{\sqrt{s}}{M_{X}} x\right)}{16 M_{X}^{5} x\left[K_{2}(x)\right]^{2}}\right]$,

where $M_{P} \approx 1.22 \times 10^{19} \mathrm{GeV}$ is the Planck mass, $g_{*} \approx 100$ is the total number of effective relativistic degrees of freedom at the time of freeze-out, and $x_{f} \approx 25$ is defined by $M_{X} / T_{f}$ at the freeze-out temperature $\left(T_{f}\right)$. Then one has

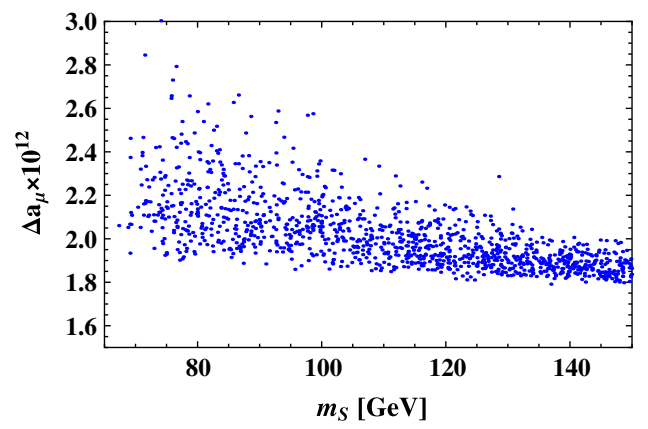

FIG. 4. Scattering plots on the muon $g-2$ and $m_{S}$ plane, where each point satisfies experimental constraints.

to satisfy the current relic density of DM: $\Omega h^{2} \approx 0.12$ [58]. In Fig. 3, we show the line of $\Omega h^{2}$ in terms of $M_{X}$, where we have used the maximum value in Eq. (2.36): $\lambda_{X X h_{1}}^{\mathrm{Max}}=0.01$. Thus, one finds that the resulting allowed region is

$54 \mathrm{GeV} \lesssim M_{X} \lesssim 62.5 \mathrm{GeV}, \quad$ for $\lambda_{X X h_{1}} \lesssim 0.01$

where the upper bound of the DM mass, $62.5 \mathrm{GeV}$, arises from the pole mass of the half SM Higgs.

\section{NUMERICAL ANALYSIS}

In this section, we show a global analysis. Before the numerical analysis, we work on the diagonal basis of $g_{L}$ by the phase redefinition of $E_{L} ; g_{L}=\operatorname{Diag}\left(g_{L_{1}}, g_{L_{2}}, g_{L_{3}}\right)$. Then we directly solve the couplings $\kappa_{1,2}$ and $g_{L_{1,2}}$ by using the relations $\left|V_{\ell_{R}} m_{\ell} V_{\ell_{L}}^{\dagger}\right|_{11(22)}=\left|m_{e(\mu)}\right|$ and $\left|m_{\nu}\right|_{11(12)} \mid \approx\left(m_{\nu}^{\exp }\right)_{11(22)}$, respectively, where we impose perturbative bounds on these output parameters: $\left(\kappa_{1,2}, g_{L_{1,2}}\right) \lesssim \sqrt{4 \pi}{ }^{4}$ Now we randomly select the following range of reduced input parameters as

$$
\begin{aligned}
M_{E} & \in[100,1000] \mathrm{GeV}, \quad m_{H_{1}^{ \pm}} \in[200,500] \mathrm{GeV}, \quad m_{H_{2}^{ \pm}} \in\left[80, M_{X}+140\right] \mathrm{GeV}, \quad m_{k^{++}} \in[500,600] \mathrm{GeV}, \\
\mu_{k h h} & \in[1,2] \mathrm{GeV}, \quad(\sin \theta, \sin \beta) \in[0, \pi / 4], \quad m_{k^{++}} \in[500,600] \mathrm{GeV}, \quad m_{S} \in\left[1.2 M_{X}, 150\right] \mathrm{GeV}, \\
\left|f_{e, \mu, \tau}, \kappa_{3}, g_{L_{3}}\right| & \in[0.001,1], \quad(\rho, \sigma) \in[0, \pi], \quad \delta \in[\pi, 2 \pi], \quad\left|s_{\alpha}\right| \in\left[10^{-5}, 0.1\right],
\end{aligned}
$$

where the lower mass range for $m_{H_{2}^{ \pm}}$arises from the bound from LEP data [59], while the upper bound is from the oblique parameters, and we impose all the constraints as discussed above.

\footnotetext{
${ }^{4}$ In principle, all the Yukawa couplings could be solved by using all the components of these relations. However, it is technically difficult in our model.
}

In Fig. 4, we show the scattering allowed plots in terms of muon $g-2$ and $m_{S}$. It suggests that the typical value of muon $g-2$ is of the order $10^{-12}$ that is smaller than the experimental value by a three-order magnitude.

In Fig. 5, we demonstrate the couplings of $\kappa_{1}-\kappa_{2}$ in the left panel, and $g_{L_{1}}-g_{L_{2}}$ in the right panel. The left one implies $2 \lesssim \kappa_{1}$ requires rather large coupling, whereas $\kappa_{2}$ is of the order 0.001 , and each of them has a weak correlation. 

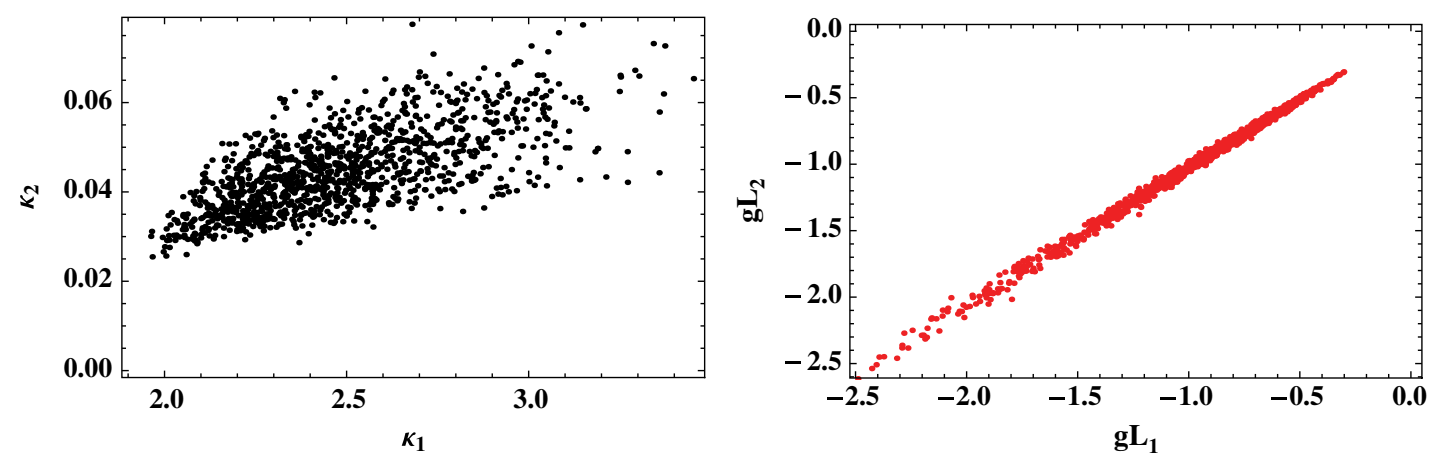

FIG. 5. Left panel: Allowed parameter points on the $k_{1}-k_{2}$ plane. Right panel: Allowed parameter points on the $g_{L_{1}}-g_{L_{2}}$ plane.

While the right one suggests both of couplings run $-2.5 \sim$ -0.3 with degeneracy to some extent.

\section{CONCLUSION}

We have constructed a radiative neutrino mass model based on a gauged lepton flavor symmetry $U(1)_{L}$. The condition needed to cancel gauge anomalies is discussed by introducing some exotic leptons with general $U(1)_{L}$ charge. Then we discussed the phenomenology of the model by fixing the charge assignment.

The neutrino mass matrix can be induced at two-loop level where the exotic leptons and charged scalar bosons propagate inside the loop diagram. On the other hand, the first and second charged leptons of SM are induced at oneloop level. Due to the feature of flavor dependent symmetry, we have predicted one massless active neutrino and a bosonic dark matter candidate from an inert doublet. Calculating the relic density, we have found that an observed value can be obtained via a Higgs portal interaction with a mass range of dark matter at 56-62.5 GeV. In addition, we have also discussed lepton flavor violation and muon $g-2$ in the model.

Then we have done a global numerical analysis to satisfy all the constraints such as charged-lepton masses, neutrino mass differences its mixing, LFVs, and oblique parameters, within the range of the DM mass. Then we have found that the typical value of muon $g-2$ is of the order $10^{-12}$, which is smaller than the experimental value by a three-order magnitude. Also, we have shown the typical Yukawa couplings of $\kappa_{1,2}$ and $g_{L_{1,2}}$, and have found typical ranges and their correlations.

\section{ACKNOWLEDGMENTS}

H. O. is sincerely grateful for the KIAS member's comments and all around support.
[1] R. Aaij et al. (LHCb Collaboration), Phys. Rev. Lett. 113, 151601 (2014).

[2] R. Aaij et al. (LHCb Collaboration), J. High Energy Phys. 08 (2017) 055.

[3] A. M. Baldini et al. (MEG Collaboration), Eur. Phys. J. C 76, 434 (2016).

[4] G. W. Bennett et al. (Muon g-2 Collaboration), Phys. Rev. D 73, 072003 (2006).

[5] A. Crivellin, J. Fuentes-Martin, A. Greljo, and G. Isidori, Phys. Lett. B 766, 77 (2017).

[6] A. Zee, Nucl. Phys. B264, 99 (1986); K. S. Babu, Phys. Lett. B 203, 132 (1988).

[7] K. S. Babu and C. Macesanu, Phys. Rev. D 67, 073010 (2003).

[8] D. Aristizabal Sierra and M. Hirsch, J. High Energy Phys. 12 (2006) 052.

[9] M. Nebot, J. F. Oliver, D. Palao, and A. Santamaria, Phys. Rev. D 77, 093013 (2008).
[10] D. Schmidt, T. Schwetz, and H. Zhang, Nucl. Phys. B885, 524 (2014).

[11] J. Herrero-Garcia, M. Nebot, N. Rius, and A. Santamaria, Nucl. Phys. B885, 542 (2014).

[12] H. N. Long and V. V. Vien, Int. J. Mod. Phys. A 29, 1450072 (2014).

[13] V. Van Vien, H. N. Long, and P. N. Thu, arXiv:1407 .8286 .

[14] M. Aoki, S. Kanemura, T. Shindou, and K. Yagyu, J. High Energy Phys. 07 (2010) 084; 11 (2010) 49.

[15] M. Lindner, D. Schmidt, and T. Schwetz, Phys. Lett. B 705, 324 (2011).

[16] S. Baek, P. Ko, H. Okada, and E. Senaha, J. High Energy Phys. 09 (2014) 153.

[17] M. Aoki, J. Kubo, and H. Takano, Phys. Rev. D 87, 116001 (2013).

[18] Y. Kajiyama, H. Okada, and K. Yagyu, Nucl. Phys. B874, 198 (2013). 
[19] Y. Kajiyama, H. Okada, and T. Toma, Phys. Rev. D 88, 015029 (2013).

[20] S. Baek, H. Okada, and T. Toma, J. Cosmol. Astropart. Phys. 06 (2014) 027.

[21] H. Okada, arXiv:1404.0280.

[22] H. Okada, T. Toma, and K. Yagyu, Phys. Rev. D 90, 095005 (2014).

[23] H. Okada, arXiv:1503.04557.

[24] C. Q. Geng and L. H. Tsai, Ann. Phys. (Amsterdam) 365, 210 (2016).

[25] S. Kashiwase, H. Okada, Y. Orikasa, and T. Toma, Int. J. Mod. Phys. A 31, 1650121 (2016).

[26] M. Aoki and T. Toma, J. Cosmol. Astropart. Phys. 09 (2014) 016.

[27] S. Baek, H. Okada, and T. Toma, Phys. Lett. B 732, 85 (2014).

[28] H. Okada and Y. Orikasa, Phys. Rev. D 93, 013008 (2016).

[29] D. Aristizabal Sierra, A. Degee, L. Dorame, and M. Hirsch, J. High Energy Phys. 03 (2015) 040.

[30] T. Nomura and H. Okada, Phys. Lett. B 756, 295 (2016).

[31] T. Nomura, H. Okada, and Y. Orikasa, Eur. Phys. J. C 77, 103 (2017).

[32] C. Bonilla, E. Ma, E. Peinado, and J. W. F. Valle, Phys. Lett. B 762, 214 (2016).

[33] M. Kohda, H. Sugiyama, and K. Tsumura, Phys. Lett. B 718, 1436 (2013).

[34] B. Dasgupta, E. Ma, and K. Tsumura, Phys. Rev. D 89, 041702 (2014).

[35] T. Nomura and H. Okada, Phys. Rev. D 94, 075021 (2016).

[36] T. Nomura and H. Okada, Phys. Rev. D 94, 093006 (2016).

[37] T. Nomura, H. Okada, and Y. Orikasa, Phys. Rev. D 94, 115018 (2016).

[38] Z. Liu and P. H. Gu, Nucl. Phys. B915, 206 (2017).

[39] C. Simoes and D. Wegman, J. High Energy Phys. 04 (2017) 148.
[40] S. Baek, H. Okada, and Y. Orikasa, arXiv:1703.00685.

[41] S. Y. Ho, T. Toma, and K. Tsumura, J. High Energy Phys. 07 (2017) 101.

[42] T. Nomura and H. Okada, arXiv:1706.01321.

[43] S. Y. Guo, Z. L. Han, B. Li, Y. Liao, and X. D. Ma, Nucl. Phys. B928, 435 (2018).

[44] F. del Aguila, A. Aparici, S. Bhattacharya, A. Santamaria, and J. Wudka, J. High Energy Phys. 05 (2012) 133.

[45] T. Nomura and H. Okada, Phys. Lett. B 761, 190 (2016).

[46] T. Nomura and H. Okada, Phys. Rev. D 96, 015016 (2017).

[47] D. V. Forero, M. Tortola, and J. W. F. Valle, Phys. Rev. D 90, 093006 (2014).

[48] K. Hagiwara, R. Liao, A. D. Martin, D. Nomura, and T. Teubner, J. Phys. G 38, 085003 (2011).

[49] T. Nomura and H. Okada, Phys. Rev. D 97, 015015 (2018).

[50] J. Adam et al. (MEG Collaboration), Phys. Rev. Lett. 110, 201801 (2013).

[51] R. Barbieri, L. J. Hall, and V. S. Rychkov, Phys. Rev. D 74, 015007 (2006).

[52] K. Cheung, T. Nomura, and H. Okada, Phys. Lett. B 768, 359 (2017).

[53] T. Hambye, F.-S. Ling, L. Lopez Honorez, and J. Rocher, J. High Energy Phys. 07 (2009) 090; 05 (2010) 66.

[54] D. S. Akerib et al. (LUX Collaboration), Phys. Rev. Lett. 118, 021303 (2017).

[55] A. Das, T. Nomura, H. Okada, and S. Roy, Phys. Rev. D 96, 075001 (2017).

[56] J. Edsjo and P. Gondolo, Phys. Rev. D 56, 1879 (1997).

[57] K. Nishiwaki, H. Okada, and Y. Orikasa, Phys. Rev. D 92 , 093013 (2015).

[58] P. A. R. Ade et al. (Planck Collaboration), Astron. Astrophys. 571, A16 (2014).

[59] G. Abbiendi et al. (OPAL Collaboration), Eur. Phys. J. C 72, 2076 (2012). 